\title{
Möglichkeiten und Grenzen einer Leistungsdefinition und individuellen Leistungsbemessung im Kontext Langzeitpflege
}

Heinz Rothgang, Thomas Kalwitzki und Janet Cordes

\section{$6.1 \quad$ Einleitung -98}

6.2 Möglichkeiten und Grenzen einer Leistungsdefinition - 99

6.2.1 Modularisierung der Pflegeleistungen - 100

6.2.2 Sektorenfreie Verpreisung der Module bei formeller Pflege - 102

6.2.3 Einbindung der Zivilgesellschaft: Pflegegeld 2.0 - 103

6.3 Institutionelle Ausgestaltung: Von der individuellen Bedarfsfeststellung zum Versorgungsarrangement - 105

6.3.1 Erste Instanz: Individuelle Bedarfsfeststellung - 105

6.3.2 Zweite Instanz: Individueller Pflegeplan, Case- und CareManagement - 105

6.3.3 Dritte Instanz: Erbringung der Pflegeleistungen - 106

6.4 Fazit -106

$$
\text { Literatur - } 108
$$




\section{- Zusammenfassung}

Die Ausgestaltung der Pflegeversicherung als nicht bedarfsdeckendes Teilleistungssystem steht zunehmend unter Reformdruck. Die pauschalierte Leistungsgewährung führt dazu, dass im stationären Sektor eine wachsende Lücke zwischen pflegebedingten Kosten und den Leistungen der Pflegeversicherung entsteht und im ambulanten Sektor aus dem gleichen Grund von einer kompensatorisch eingeschränkten Leistungsinanspruchnahme auszugehen ist. Beide Effekte werden zwar zu Recht als Problem des Umfangs der Versicherungsleistungen dargestellt, diese sind jedoch letztendlich Konsequenz einer fehlenden individuellen Bedarfsorientierung.

Die Autoren schlagen daher vor, eine Finanzreform der Pflegeversicherung direkt mit einer Strukturreform zu verbinden, die eine bedarfsorientierte Pflege in jeder Wohnform ermöglicht. Hierzu ist es erforderlich, die übernahmefähigen Leistungen/Leistungsbereiche der Pflegeversicherung unabhängig vom Ort der Erbringung $z u$ definieren und mit einem einheitlichen Preisschema zu hinterlegen. Anhand dieses Kataloges wird es möglich, die individuell bedarfsorientierte Leistungsmenge zu bemessen und den Pflegebedürftigen zuzuordnen. Dies kann zudem genutzt werden, um das Pflegegeld des heutigen Zuschnitts zu einem Cash-for-Care-System für die Pflegepersonen weiterzuentwickeln.

The German long-term care insurance is based on a system of capped benefits per care grade, as opposed to the care recipient's individual level of need and ability to pay. This has led to a growing role for out-of-pocket payments in nursing home care, as well as insufficient in-kind benefits in home care. Both effects are an effect of the system's inability to secure a needs-based approach to care.

In order to address these problems in longterm care, the authors argue for a combined financial and structural reform of the German system. To achieve this, a uniform catalogue of services must be introduced across care settings using a shared pricing scheme. This can serve as the basis for a future individually oriented, needs-based system of long-term care provision.

\subsection{Einleitung}

Nach einem Vierteljahrhundert ihrer Existenz ist die Pflegeversicherung sowohl eine sozialpolitische Erfolgsgeschichte als gleichzeitig auch ein Dauerpatient mit vielen Reformbedarfen. Diese entstehen oder verstärken sich zum einen durch die Veränderung der äußeren Rahmenbedingungen. Das lässt sich besonders im Bereich von Personal und Personalbedarf erkennen, in dem durch die Alterung der Bevölkerung ein doppelter demographischer Effekt entsteht: Während immer mehr ältere und pflegebedürftige Menschen zu einem immer größeren Pflegebedarf führen, steht für die berufliche Pflege eine tendenziell abnehmende Zahl von Erwerbstätigen zur Verfügung, sodass sich als „Pflegenotstand“ bezeichnete personelle Versorgungslücken zeigen. Dem entgegenwirkende Reformen werden vor allem in der Ausbildung, der Rekrutierung und in der Attraktivitätsförderung des Pflegeberufs umgesetzt. Zum anderen entstehen Reformbedarfe allerdings auch von innen aufgrund zweier Ausgestaltungsregelungen der Pflegeversicherung: der Ausgestaltung als pauschaliertes Teilleistungssystem mit seiner - sukzessive genutzten Möglichkeit zu unbegrenzter privater Kostenbeteiligung bei unzureichender Anpassung der Pauschalen und der rechtlich tief verwurzelten Fragmentierung in einen ambulanten und einen stationären Sektor. Während die unzureichenden Pauschalen dabei die Bedarfsorientierung in quantitativer Hinsicht vernachlässigen, erschwert die Fragmentierung es, qualitativ bedarfsgerechte Versorgungsstrukturen zu schaffen. Beide Regelungen sind als Geburtsfehler anzusehen, die zur politischen Durchsetzung der Pflegeversicherung Anfang der 1990er Jahre erforderlich waren. Allerdings sind ihre Auswirkungen durch sukzessive Nachsteuerungen nicht mehr hinreichend zu begrenzen. Vielmehr verhindern sie mittlerweile weitgehend das coming of age zu einer stabilen Sozialversicherung, bei der nicht in immer kürzeren Zeitabständen Reformschritte notwendig werden. 
Bereits seit einigen Jahren beschäftigen sich die Autoren dieses Artikels intensiv mit den aktuellen Reformerfordernissen, denen sich die Pflegeversicherung gegenübersieht. Als Lösungsweg wurde dabei eine integrierte Finanzund Strukturreform für die Pflegeversicherung ausgearbeitet und veröffentlicht (Rothgang und Kalwitzki 2017; Rothgang et al. 2019, 2020). Die Reformkonzeption beinhaltet als Element der Finanzreform ein Konzept, das mittlerweile unter dem Begriff Sockel-Spitze-Tausch populär geworden ist und vordergründig auf die absolute und über Zeit stabile Begrenzung der privat zu zahlenden Eigenanteile an den pflegebedingten Kosten abzielt (vgl. Rothgang und Kalwitzki 2018, 2019). Dieser Reformschritt löst nicht nur das Problem der derzeit (zu) hohen Eigenanteile in der stationären Pflege (Rothgang 2020), sondern geht weit darüber hinaus, indem er eine bedarfsgerechte Leistungsausgestaltung konzipiert wie sie in der Krankenversicherung als selbstverständlich betrachtet wird (vgl. hierzu auch Schwinger und Tsiasioti 2020).

Gleichzeitig mit der Finanzreform wird aber auch eine Strukturreform der Pflegeversicherung vorgeschlagen, bei deren Umsetzung das Leistungsgeschehen nicht mehr anhand der Grenzen eines ambulanten und eines stationären Sektors organisiert, sondern lediglich eine Grenzlinie zwischen den (Dienst)Leistungsbereichen Wohnen und Pflege gezogen wird, Pflegeleistungen aber sektorenfrei organisiert werden. Dies impliziert einen Perspektivwechsel, nach dem die Inhalte der Pflegeleistungen sich nicht mehr durch den Ort der Leistungserbringung bestimmen, sondern unabhängig von diesem durch die Bedarfe des Pflegebedürftigen und die Interaktionen mit diesem bestimmt werden. Umfang und auch Art der bedarfsgerechten Leistungen sind also für jeden Pflegebedürftigen individuell unterschiedlich und müssen sowohl für die pflegerische Leistungserbringung als auch für die sozialrechtliche Leistungshöhe berücksichtigt werden.

Dabei stellt sich die Frage nach dem Verhältnis der beiden Reformelemente. Während der Sockel-Spitze-Tausch auch auf den stationären Sektor begrenzt werden kann und dann die sektorale Fragmentierung unberührt lässt so etwa im Reformmodell der DAK Gesundheit für die Pflegeversicherung (DAK 2019) - und die Schaffung einer sektorenfreien Versorgung auch ohne eine Begrenzung der Eigenanteile möglich ist, führt erst die Kombination beider Elemente zu einer individuell bedarfsgerechten Versorgung bei Kontrolle des Risikos der pflegebedingten Verarmung.

So entfaltet die individuell bedarfsgerechte Ausgestaltung der Leistungshöhen, die durch den Sockel-Spitze-Tausch eingeführt wird, ihre volle Wirksamkeit erst, wenn auch die Wahl des Wohnortes und der Wohnform keine Auswirkungen mehr auf die Eigenanteile an den Pflegekosten hat. Gleichzeitig können die gesteigerten Wahlmöglichkeiten, die aus der Aufhebung der Sektorengrenzen resultieren, besser genutzt werden, wenn sie von einer Finanzierungslösung flankiert werden, die durch das neue Leistungsgeschehen keine zusätzlichen Armutsrisiken schafft und gleichzeitig eine unbeabsichtigte Überinanspruchnahme von Pflegeleistungen wirksam ausschließen kann.

Voraussetzung für die Strukturreform ist dabei die Individualisierung der Leistungszumessung auf Basis der konkreten Fähigkeiten und Hilfebedarfe, die gleichzeitig eine moralhazard-bedingte Überinanspruchnahme ausschließt. Nachfolgend wird dargestellt, wie eine hierzu notwendige Leistungsdefinition vorgenommen werden kann ( Abschn. 6.2) und wie die dazu notwendigen Prozesse institutionell ausgestaltet werden können ( $\triangleright$ Abschn. 6.3). Im Fazit ( Abschn. 6.4) werden abschließend die Reformvoraussetzungen und die Möglichkeit ihrer Umsetzung zusammenfassend diskutiert.

\subsection{Möglichkeiten und Grenzen einer Leistungsdefinition}

Ziel der hier vorgestellten Strukturreform ist es, in jedem individuellen Wohnsetting ein individualisiertes Pflegearrangement für jeden 
Pflegebedürftigen $\mathrm{zu}$ organisieren, in dessen Rahmen formelle und informelle Pflege optimal zusammenwirken. In einer sektorenfreien Versorgungslandschaft ist die zentrale Frage dabei nicht mehr, wo eine Pflegeleistung erbracht wird, sondern durch wen. Ziel ist es, entsprechend der individuellen Bedarfslage und der individuellen Präferenzen ein je spezifisches Pflegearrangement zu ermöglichen, das eine bedarfsgerechte Leistungsmenge enthält, die durch das Zusammenspiel formeller und informeller Pflegepersonen verbindlich erbracht wird. Durch die Beseitigung der sektoralen Fragmentierung entsteht so ein Raum, in dem kreative, innovative Versorgungsangebote ermöglicht werden und in dem Versorgungsstrukturen nicht mehr in erster Linie durch die ordnungsrechtlichen Einschränkungen der sektoralen Anforderungen strukturiert werden. Durch diese Neustrukturierung wird es dann möglich, die bisher bestehende Vollversorgungspflicht in (vormals) stationären Einrichtungen aufzuheben und es wird die Voraussetzung dafür geschaffen, die Leistungen der Zivilgesellschaft in das individuelle Pflegearrangement auch in derartigen Wohnarrangements mit aufzunehmen.

Um eine sektorenfreie Versorgungslandschaft zu ermöglichen, müssen Leistungen inhaltlich in Form von Leistungsmodulen beschrieben werden. Dabei muss diese Leistungsbeschreibung vom Ort der Leistungserbringung abstrahieren $(\triangleright$ Abschn. 6.2.1). Für diese Leistungen müssen dann Entgelte festgelegt werden, die an formelle Pflegeanbieter zu zahlen sind ( $\triangleright$ Abschn. 6.2.2), und es muss geklärt werden, welche Zahlungsströme ausgelöst werden, wenn diese Leistungen informell erbracht werden ( $\triangleright$ Abschn. 6.2.3).

\subsubsection{Modularisierung der Pflegeleistungen}

Die praktischen Anforderungen an ein modularisiertes und vom Ort der Erbringung unabhängiges Leistungsgeschehen sind umfang- reich. Einerseits müssen die Module hinreichend groß sein, um Abgrenzungsprobleme zu minimieren. Andererseits muss die Granulation des Leistungsgeschehens so fein sein, dass eine Übernahme genau umrissener Leistungsteile durch zivilgesellschaftliche Akteure auch in kleinerem Umfang praktikabel ist. $\mathrm{Da}$ die Sektorentrennung aufgehoben werden soll, sind diese Module zudem so zu konzipieren, dass sie in verschiedenen Wohnsettings einheitlich angeboten werden können. Bisher bestehende Ungleichbehandlungen etwa von hauswirtschaftlichen Leistungen in ambulanter und stationärer Versorgung müssen dabei aufgehoben werden. Letztlich ist es also erforderlich, einen abschließenden Modul- und Leistungskatalog auszuarbeiten, in dem alle Leistungen, die im Rahmen einer Leistungszumessung als bedarfsgerecht im Sinne der Pflegeversicherung gelten können, überschneidungsfrei abgebildet sind. Für einen solchen Leistungskatalog, der zur Erstellung einer individuellen Pflegeplanung wohnortunabhängig genutzt werden kann, ergeben sich dabei folgende Anforderungen:

- Die Definition der Leistungen muss unabhängig vom Ort der Leistungserbringung erfolgen.

- Der Katalog muss umfassend sein und alle Leistungen einschließen, die im Bereich des SGB XI übernommen werden können.

- Für den Bereich der Langzeitpflege müssen die Leistungen so definiert sein, dass sie - mit Ausnahme von Steuerungs- und Regieleistungen - sowohl von professionellen Pflegekräften als auch von Laienkräften - nach spezifischer Schulung oder laufender Anleitung - erbracht werden können. Entsprechend muss ein Modul enthalten sein, das Leistungen beinhaltet, die zur Steuerung der Pflege erforderlich sind.

- Individual- und Gruppenleistungen müssen unterschieden werden, da die Finanzierung von Gruppenleistungen nur anteilig erfolgen soll.

- Der Modul- und Leistungskatalog muss praktikabel sein und die Anreize für die 
Leistungsübernahme durch die Zivilgesellschaft stärken.

- Medizinisch-therapeutische und medizinisch-diagnostische Leistungen, die im Bereich des SGB V übernommen werden und nur von beruflich Pflegenden übernommen werden können, müssen als separates Modul ausgewiesen werden.

Zur Herleitung eines entsprechenden Modulund Leistungskataloges hat sich die Recherche der internationalen Literatur als nicht zielführend erwiesen. Dazu wurde in einer internationalen Fachdatenbank (Pubmed) und im Rahmen einer Webrecherche bei Google Scholar zu Leistungskatalogen und Leistungszumessung auf internationaler Ebene recherchiert. Im Ergebnis standen Übersichtsarbeiten und Vergleichsstudien zu internationalen Pflegesystemen, in denen eine detailliertere Darstellung von Leistungskatalogen oder Leistungszumessung nicht zu finden war (Spasova et al. 2018; Heintze 2015; TRISAN 2018; Geyer et al. 2016). Die Recherche im landesrechtlichen Kontext einzelner Länder (Dänemark, Schweden, Niederlande) war ebenfalls ergebnislos. Hauptursache hierfür ist, dass eine Leistungssystematik, die Tätigkeiten kleinschrittig definiert, um so einen Anreiz zur zivilgesellschaftlichen Übernahme zu schaffen, in anderen Ländern nicht vorgesehen ist. Die Ausgestaltung von Einzelinterventionen ist in dieser Form in den untersuchten Ländern nicht etabliert. Um den oben genannten Anforderungen wie der Trennung von Pflege und Wohnen und der Leistungsübernahme durch informelle Pflegepersonen auch in institutioneller Wohnform zu entsprechen, ist eine feine Granulierung der Einzelleistungen allerdings unabdingbar. Für die Erstellung des Leistungskataloges wurden daher neben pflegefachlicher Literatur (Bartoszek und Drude 2015; Bulechek et al. 2016; Kirschnick 2016) bestehende Leistungskataloge berücksichtigt, die das vollständige Leistungsgeschehen beschreiben und die Ganzheitlichkeit der pflegerischen Versorgung abbilden. Für den ambulanten Bereich konnten die ambulanten Leistungskataloge der Bundesländer einbezogen werden. Für den stationären Bereich gibt es einen solchen Katalog nicht, da im Sinne einer Vollversorgung keine Erbringung auf Leistungsebene erfolgt. Aus diesem Grund wurde der Modul- und Leistungskatalog herangezogen, der im Rahmen der Studie Evaluation des NBA - Erfassung von Versorgungsaufwänden in stationären Einrichtungen (EViS) (Rothgang et al. 2015) entwickelt wurde und das gesamte Leistungsspektrum der vollstationären Versorgung in den Blick nimmt. Zusätzlich wurden Ergänzungen vorgenommen, die aus dem Abgleich der Erkenntnisse einer Recherche pflegefachlicher Literatur und dem Interventionskatalog des Projektes nach $\$ 113 \mathrm{c}$ SGB XI zur Personalbemessung in Einrichtungen der Langzeitpflege (PeBeM) ermittelt werden konnten. Der PeBeM-Interventionskatalog wurde zur Erstellung einer individuellen Pflegeplanung in (teil-)stationären Pflegeeinrichtungen ähnlich einem Case-Management genutzt und bildet ebenfalls das vollständige Leistungsgeschehen in stationären Pflegeeinrichtungen ab. Wo der EViS-Katalog teilweise sehr feine Aufsplitterungen der Leistungen enthält, konnte der PeBeM-Katalog einzelne Leistungen durch Leitungskomplexe ganzheitlicher darstellen. Die ambulanten Leistungskataloge umfassen dagegen detaillierter hauswirtschaftliche Leistungen, die in stationären Katalogen aufgrund der leistungsrechtlichen Zuordnung eher einen geringeren Stellenwert einnehmen. Um den neuen Pflegebedürftigkeitsbegriff und das ihm zugrunde liegende Pflegeverständnis zu integrieren, wurden die Strukturierung und Beschreibung pflegerischer Aufgaben auf der Grundlage des neuen Pflegebedürftigkeitsbegriffs (Wingenfeld und Büscher 2017) konzipiert.

Mittels dieser Herangehensweise ist ein Modul- und Leistungskatalog entstanden, der insgesamt 40 Leistungen des SGB XI enthält, die den Modulen „Pflege und Betreuung“, „Hilfe bei der Haushaltsführung“ und „Steuerung der Pflege“ zugeordnet wurden. Im Modul „Pflege und Betreuung“ sind dabei 22 Leistungen enthalten, die den Bereichen Mobilität, pflegerische Betreuung und Selbstversor- 
gung zugeordnet wurden. Das Modul „Hilfe bei der Haushaltsführung" umfasst acht hauswirtschaftliche Leistungen und das Modul „Steuerung der Pflege“ enthält zehn Leistungen, die zur Pflegeprozessteuerung und Qualitätssicherung bei zivilgesellschaftlicher Übernahme erforderlich sind. Insgesamt 23 Einzelleistungen, die dem Modul „Hilfe bei der Bewältigung von krankheits- und therapiebedingten Aufgaben" zugeordnet sind, umfassen Leistungen, die derzeit als medizinische Behandlungspflege (in stationärer Pflege) bzw. häusliche Krankenpflege (bei häuslicher Pflege) verordnet werden (s. Rothgang et al. 2019, S. 44 ff. für Details).

Dieser Modul- und Leistungskatalog ist dabei nicht als finales Instrument zu verstehen, sondern im Sinne eines proof of concept als Nachweis dafür, dass eine Modularisierung des Leistungsgeschehens bei Beachtung der genannten Anforderungen möglich ist. Selbstverständlich ist eine Weiterentwicklung dieses Katalogs im Zuge der Umsetzung der Strukturreform nicht nur denkbar, sondern auch wünschenswert.

\subsubsection{Sektorenfreie Verpreisung der Module bei formeller Pflege}

Für die Erbringung der Module durch formelle Pflegekräfte sind Entgelte festzulegen. Entscheidend ist, dass dabei ein einheitlicher sektorenfreier Modulkatalog verwendet wird und dass für diese Module wohnortunabhängige Preise festgelegt werden. Etwaige Wegezeitvergütungen werden dabei gesondert als eigene Leistung bepreist und abgerechnet.

Für jede Leistung wird zunächst eine Punktzahl festgelegt. Die Punktrelationen zweier Leistungen sind dabei Ausdruck des Aufwandsverhältnisses, in volkswirtschaftlicher Terminologie: relative Preise. Diese Punktrelationen können bundeseinheitlich festgelegt werden - wie dies beim einheitlichen Bewertungsmaßstab für ambulante ärztliche Leistungen oder auch bei den Fallgewichten im Rahmen der DRG-basierten Fallpauschalenvergütung in Krankenhäusern geschieht.

Da sich die Versorgungsaufwände bei gleicher Leistung zwischen Pflegebedürftigen mit unterschiedlichen Schweregraden der Pflegebedürftigkeit unterscheiden, können je nach Pflegegrad - oder nach einem noch weiter differenzierten Klassifikationssystem für Pflegebedürftige - Hebesätze vereinbart werden, die diesem Schweregrad Rechnung tragen. Damit hat der Zustand der Pflegebedürftigen nicht nur einen Einfluss auf die Mengenkomponente der Leistungserbringung, sondern auch auf deren Zeitkomponente und könnte daher in die Preissetzung mit einbezogen werden. Solche Hebesätze können direkt von den Merkmalen abhängig gemacht werden, mit denen die pflegebedürftige Person im Rahmen des Begutachtungsinstruments charakterisiert wird, da diese - wie sich im Rahmen der Entwicklung und Erprobung eines Personalbemessungsverfahrens nach $₫ 113$ c SGB XI gezeigt hat - den Aufwand gut vorhersagen können (Rothgang et al. 2020, S. 269 ff.).

Auch in der Pflege gibt es typische Gemeinkosten, wie die Leistungen des Qualitäts- oder Personalmanagements, die nicht im Modulund Leistungskatalog hinterlegt sind, da es nicht sinnvoll erscheint, diese Leistungen auf Individualebene zu planen. Vielmehr sind mit diesen Leistungen Beschäftigtengruppen verbunden, die für die Aufrechterhaltung einer professionellen Institution notwendig sind. Um diese Gruppe bei der Preissetzung einzubeziehen, ist ein Gemeinkostenfaktor vorzusehen, mit dem die jeweiligen Punktzahlen multipliziert werden müssen.

Um zu Euro-Beträgen zu gelangen, müssen die jeweiligen Punktzahlen mit einem Punktwert multipliziert werden, der in Euro pro Punkt angegeben wird. Die Punktwerte können bundes- oder landesweit festgelegt werden. Zur Berücksichtigung der derzeitigen unterschiedlichen Preisniveaus erscheint eine Festsetzung der Punktwerte in einem Verhandlungssystem auf Landesebene derzeit zielführend, allerdings mit der Perspektive, im Rahmen eines Konver- 
genzprozesses zu einer Angleichung der landesspezifischen Punktwerte zu gelangen. Unterschiedliche Preise je nach Trägerschaft der Einrichtung sind dagegen nicht vorgesehen. Die Grundlage hierfür entfällt zudem in dem Maße, in dem einheitliche Tarifverträge an Geltung gewinnen und die Personalausstattung von Einrichtungen über ein Personalbemessungsverfahren angeglichen wird.

Bislang ist in Pflegeheimen die Abgrenzung der pflegebedingten Aufwände und der Aufwände für Unterkunft und Verpflegung in den Bundesländern uneinheitlich geregelt (Augurzky et al. 2008, S. 26 f.). Dies ist derzeit insofern unerheblich, als die Pflegebedürftigen sowohl Veränderungen beim einen als auch beim anderen Aufwand jeweils zu $100 \%$ selbst übernehmen müssen. Werden die Pflegekosten dagegen nach dem Sockel-Spitze-Tausch von der Pflegeversicherung übernommen, gewinnt diese Abgrenzung an Bedeutung und muss bundesweit einheitlich geregelt werden.

Gleichermaßen unterschiedlich ist die Behandlung hauswirtschaftlicher Aufwände im Vergleich von derzeit ambulanter und stationärer Leistungserbringung: Im stationären Sektor werden diese Aufwände dem Bereich Unterkunft und Verpflegung zugeordnet und sind vom Pflegebedürftigen zu zahlen. Im ambulanten Bereich können entsprechende Leistungsmodule dagegen auch heute schon zu Lasten der Pflegeversicherung erbracht werden. Bei einer sektorenfreien Versorgung muss auch hier eine einheitliche Regelung getroffen werden. Die Entgelte für Unterkunft und Verpflegung im Heim übersteigen die entsprechenden Aufwände in privaten Haushalten derzeit bereits beträchtlich - nicht zuletzt, weil ein Teil dieser Aufwände "pflegebedingt" ist. Es wird daher davon ausgegangen, dass bei einer einheitlichen Grenzziehung ein Teil der derzeit im Heim der Unterkunft und Verpflegung zugerechneten Aufwände als Pflegekosten eingestuft werden müssen. Im Modul- und Leistungskatalog wurden diese Leistungen ebenfalls vorerst dem SBG XI zugeordnet.

Eine weitere Herausforderung bei der Entwicklung eines bundesweiten Preissys- tems stellt neben der hohen Diversität der Preissetzungen der Leistungen auch die Leistungsabrechnung dar. Zur Abrechnung der einzelnen Leistungen sind optimierte Lösungen notwendig, die bisher in dieser Form nicht vorhanden sind. So impliziert die Abrechnung von SGB-V-Leistungen für ambulante Pflegedienste derzeit einen hohen administrativen Aufwand. Digitalisierungsprozesse, wie sie bereits durch die Konzertierte Aktion Pflege (BMG 2019) in den Pflegeeinrichtungen angestoßen sind, bieten die Möglichkeit, entsprechende Softwareprogramme zu entwickeln und flächendeckend in den Pflegemarkt zu integrieren, um so die Abrechnung von SGB Vund SGB XI-Leistungen gleichermaßen zu optimieren.

\subsubsection{Einbindung der Zivilgesellschaft: Pflegegeld 2.0}

Werden die Module durch informelle Pflegepersonen erbracht, so sollten diese dafür eine finanzielle Zuwendung erhalten. Dabei muss festgelegt werden, wie diese Zuwendung erfolgt, welche Voraussetzungen dafür gelten und welche Zuwendungshöhen hier anzusetzen sind.

Einer konkreten und überprüfbaren Zweckbindung unterliegt das Pflegegeld derzeit nicht. Wie die Repräsentativbefragung von Kantar zeigt, gaben 2016 nur $59 \%$ der Befragten das Pflegegeld teilweise oder ganz als Aufwandsentschädigung für private Hauptpflegepersonen in der Familie oder für sonstige pflegende Angehörige oder Bekannte weiter. $28 \%$ der Befragten benötigen es dagegen ganz oder teilweise für laufende Ausgaben zum Lebensunterhalt und $13 \%$ für Sonstiges (Mehrfachantworten waren möglich) (Schneekloth et al. 2017, S. 81). Da das Pflegegeld u. a. auch zur Deckung der durch die Pflege entstehenden laufenden Ausgaben und für Sachleistungen, die in der Pflegeversicherung nicht vorgesehen sind, verwendet wird (ebd.), kann davon ausge- 
gangen werden, dass weniger als die Hälfte der Pflegegeldzahlungen bei Hauptpflegepersonen ankommt.

Im vorliegenden Reformvorschlag soll das Pflegegeld dagegen zu einer Regelleistung für die Pflegepersonen weiterentwickelt werden und dabei nicht hinter das aktuelle Niveau zurückfallen. Das neue „Pflegegeld 2.0“ ist durch folgende Merkmale gekennzeichnet: Das Pflegegeld wird

- weiterhin steuer- und beitragsfrei,

- nunmehr aber an die Pflegeperson und nicht mehr an den Pflegebedürftigen ausgezahlt - wie dies in einer Reihe europäischer Staaten, die Cash-for-Care-Leistungen vorsehen, bereits üblich ist (Gori und Morciano 2019),

- unabhängig vom Ort der Leistungserbringung gewährt, und zwar

- nur, wenn im Gegenzug die Erbringung bestimmter als bedarfsnotwendig anerkannter Pflegeleistungen zugesagt wird.

Auf Seiten der Pflegepersonen wird die Auszahlung des weiterentwickelten Pflegegeldes nun also an eine Leistungsverpflichtung geknüpft, und auch private Pflegepersonen werden im Rahmen der Ausgestaltung eines individuellen Pflegearrangements auf genau definierte Leistungserbringungen kontrahiert. Damit besteht die Möglichkeit, sie auf eine regelmäßige und qualitativ angemessene Leistungserbringung und auf den Erwerb einer Basisqualifikation, auf Inanspruchnahme einer leistungsbezogenen Anleitung und auf eine Qualitätsprüfung zu verpflichten.

Die Höhe des Pflegegeldes entspricht $40 \%$ des Wertes, den ein Anbieter professioneller Pflege für die jeweilige Leistung erhalten würde. Der Anteilswert von $40 \%$ lehnt sich an das derzeitige Verhältnis von Pflegegeld zu Sachleistungen an. Er stellt zudem sicher, dass Pflegehaushalte, die alle Module selbst erbringen, bei einem angesetzten durchschnittlichen Pflegebedarf auch nach Abzug eines Sockels von $471 €$ im Jahr 2020 im Durchschnitt Leistungen der Pflegeversicherung erhalten, die nicht unterhalb der derzeitigen maximalen Pflegegeldleistungen liegen.

Zur vollständigen Integration der zivilgesellschaftlichen Leistungserbringer als verlässlichen Teil der individuellen Pflegearrangements sind drei wesentliche strukturelle Anforderungen zu beachten und umzusetzen:

Erstens muss die Grundlage dafür geschaffen werden, dass die zivilgesellschaftlichen Akteure auf ihren jeweiligen Teil der Erbringungsverantwortung verpflichtet werden können. Dazu sind Kontrahierungsinstanzen erforderlich, denen der Abschluss rechtlich bindender Vertragskonstellationen zwischen Laien und Pflegekassen übertragen werden kann. Da im individualisierten Pflegearrangement keine übergeordnete Gesamterbringungsverantwortung der Leistungserbringer mehr existiert, muss diese Funktion institutionell übernommen werden.

Zweitens muss eine Struktur geschaffen werden, durch die die zivilgesellschaftlichen Leistungserbringer entsprechend ihrer übernommenen Tätigkeiten eine verpflichtende Qualifikation erhalten. Sie sollen - analog zur Kindertagespflege - eine Grundqualifikation und eine leistungsbezogene Anleitung als Voraussetzung für die Übernahme bestimmter Leistungen verbindlich nachzuweisen haben.

Drittens muss diese quantitative und qualitative Leistungsverpflichtung mit einem Prüfund ggf. Sanktionsmechanismus hinterlegt werden. Auch wenn es systematisch sinnvoll erscheint, für einen endgültigen Vorschlag auf die abschließende Ausarbeitung der ambulanten Qualitätsindikatoren und ihre Umsetzung $\mathrm{zu}$ warten, wird eine entsprechende institutionelle Verankerung bereits im Folgenden mit vorgestellt. 


\subsection{Institutionelle Ausgestaltung: Von der individuellen Bedarfsfeststellung zum Versorgungsarrangement}

Die Umsetzung des Reformmodells erfordert nicht nur die inhaltliche Definition von Leistungsmodulen, sondern auch Regelungen über die institutionelle Ausgestaltung, also Antworten auf die Frage, wer worüber entscheidet. Dabei sind zunächst strukturelle Entscheidungen auf der Makroebene zu treffen. Hierzu zählt die Festlegung eines Modul- und Leistungskatalogs, die relativen Preise für einzelne Leistungen in Form von Punktzahlen und der Punktwert, mit dessen Hilfe Punktzahlen in EuroBeträge transformiert werden. Diese Entscheidungen sind auf politischer Ebene bzw. in den Gremien der gemeinsamen Selbstverwaltung zu treffen. Innerhalb des so vorgegebenen Rahmens ist auf Mikroebene für jede einzelne pflegebedürftige Person ein individuelles bedarfsgerechtes Pflegearrangement zu identifizieren und $\mathrm{zu}$ organisieren. Dieser Prozess kann in drei Schritten konzipiert werden, wobei für jeden Schritt eine eigene Entscheidungsinstanz vorgesehen ist.

\subsubsection{Erste Instanz: Individuelle Bedarfsfeststellung}

Im ersten Schritt ist eine Bedarfsfeststellung vorzunehmen. Institutionell ist hierzu eine Instanz vorzusehen, die in der Lage ist, den Pflegebedarf individuell festzustellen und ein zur Sättigung dieser Bedarfe notwendiges Budget zu ermitteln. Hierzu scheint der Medizinische Dienst in besonderer Weise prädestiniert $\mathrm{zu}$ sein. Dieser erhält - als erste Instanz - drei Aufgaben:

- Zunächst muss er nach wie vor die Pflegebedürftigkeit feststellen und - soweit er für die Ermittlung von Hebesätzen (s. o.) noch benötigt wird - den Pflegegrad. Zur Feststellung der Pflegebedürftigkeit kann der Me- dizinische Dienst auf das Begutachtungsinstrument in der vorliegenden Form (MDS und GKV 2017) zurückgreifen. Ebenfalls bleiben die bisherigen Voraussetzungen der Leistungsberechtigung nach $\$ 14$ SGB XI unangetastet.

- Auf Basis der individuellen Pflegebedürftigkeit und der konkreten Pflegesituation werden dann unter Rückgriff auf den Modul- und Leistungskatalog die individuell notwendigen Leistungsmengen abgebildet - wie dies in den Pflegesicherungssystemen anderer Länder und in Deutschland seit Inkrafttreten des Bundesteilhabegesetzes im Behindertenbereich üblich ist. Da bei der Bestimmung des Pflegegrades durch die mehrfache Klassierung im Rahmen der Bewertungssystematik Teile der erhobenen Informationen verloren gehen, sollte dabei direkt auf die 64 Items des Begutachtungsinstruments (BI) abgestellt werden, die den Pflegebedarf ganzheitlich und individuell abbilden. Die einzelnen Items des BI sind dafür besonders geeignet, da sie neben psychischen Problemlagen auch kognitive und kommunikative Fähigkeiten in einer bisher nicht vergleichbaren Form beurteilen (Wingenfeld et al. 2011, S. 66).

- Unter Berücksichtigung der erforderlichen Qualifikationsanforderungen, notwendiger Erbringungszeiten, wie sie beispielsweise auch im Projekt zur Personalbemessung nach $₫ 113$ c SGB XI erhoben wurden, und des Lohn- bzw. Entgeltniveaus für die Leistungen lässt sich hieraus ein individuell bedarfsnotwendiges Pflegebudget berechnen.

\subsubsection{Zweite Instanz: Individueller Pflegeplan, Case- und Care- Management}

In der zweiten Instanz wird in diesem Budgetrahmen auf kommunaler Ebene ein individueller Pflegeplan erarbeitet, an den sich bei komplexeren Fällen ein individuelles Case-Management anschließt. Da hierzu eine Kenntnis 
der lokalen Angebotsstrukturen unabdingbar ist, kann die individuelle Pflegeplanung nur auf kommunaler Ebene angesiedelt sein, wobei die Kommune diese Aufgaben selbst übernehmen oder an entsprechend zertifizierte Anbieter delegieren kann.

Die auf dieser Ebene verorteten Aufgaben umfassen zum einen die Erstellung eines Leistungsplans zur Steuerung und Verteilung des Budgets in Absprache mit den Pflegebedürftigen und deren Angehörigen und die Beratung über lokale professionelle Anbieter. Zum anderen wird die Übernahme der Leistungserbringung durch die professionellen Anbieter und/ oder Laien koordiniert. Bei zivilgesellschaftlicher Übernahme erfolgt hier die Kontrahierung der Erbringung konkreter Leistungsinhalte zwischen Pflegekasse und zivilgesellschaftlicher Pflegeperson. Verbleibende Budgetanteile werden vom Case-Manager entsprechend dem Leistungsplan und dem Wunsch der Pflegebedürftigen auf die professionellen Anbieter aufgeteilt. Möglich ist hier, das aufgeteilte Budget in Form von "Gutscheinen“ an die Pflegebedürftigen und deren Angehörigen auszuhändigen, mit denen diese schließlich an die professionellen Anbieter herantreten können.

\subsubsection{Dritte Instanz: Erbringung der Pflegeleistungen}

Bei der Erbringung der Leistungen fungieren die formellen Leistungserbringer nun als dritte Instanz. Die Pflegebedürftigen und deren Angehörigen können mit den „Profi-Gutscheinen“ das aufgeteilte Budget zusammen mit den professionellen Anbietern in Form eines individuellen Pflegeplanes frei und flexibel verplanen. Die professionellen Anbieter übernehmen somit das Leistungsmanagement unterhalb der kommunalen Ebene und führen regelmäßige Reflexions- und Evaluationsgespräche zur Leistungserbringung mit den pflegebedürftigen Personen und deren Angehörigen durch. Bei Veränderungen des Pflegebedarfs können sie innerhalb ihres Budgets flexibel gemein- sam Änderungen der Pflegeplanung vornehmen und bei höherem Pflegebedarf und somit Budgetüberschreitungen bei den Pflegekassen eine Neubegutachtung anregen.

Darüber hinaus übernehmen die professionellen Pflegeanbieter wie bisher auch die Qualitätssicherung der Pflege durch Angehörige, allerdings leistungsbezogen und damit konkreter als in den heutigen Regelungen des $₫ 36$ Abs. 3 SGB XI. Um eine Sicherstellung der Pflegequalität zu gewährleisten, wird für zivilgesellschaftliche Pflegepersonen eine verpflichtende Basisschulung für die kontrahierten Leistungen durchgeführt sowie eine begleitende Anleitung zu pflegerischen Tätigkeiten angeboten. Diese Aufgaben werden von professionellen Pflegeanbietern gegen entsprechende Leistungsvergütungen erbracht. - Abb. 6.1 fasst die institutionelle Ausgestaltung der Aufgaben im Strukturreformmodell noch einmal zusammen.

\subsection{Fazit}

Ausgehend von dem Befund, dass die Pflegeversicherung ein Vierteljahrhundert nach ihrer Einführung sowohl eine Finanz- als auch eine Strukturreform benötigt, wurde vorstehend ausgeführt, wie eine solche Strukturreform aussehen könnte. Dabei zeigt sich, dass diese voraussetzungsvoll ist, werden dazu doch auf der Makroebene

- ein sektorübergreifender Modul- und Leistungskatalog sowie

- ein System zur Verpreisung dieser Leistungen und

- die Umwandlung des derzeitigen Pflegegelds in ein „Pflegegeld 2.0“

ebenso wie auf der Mikroebene

- ein Verfahren zur individuellen Bedarfsfeststellung und

- zur individuellen Pflegeplanung, die bei komplexeren Fällen ein individuelles CaseManagement umschließt,

benötigt. Dabei sorgen Care-ManagementStrukturen dafür, dass die benötigten Hilfs- 


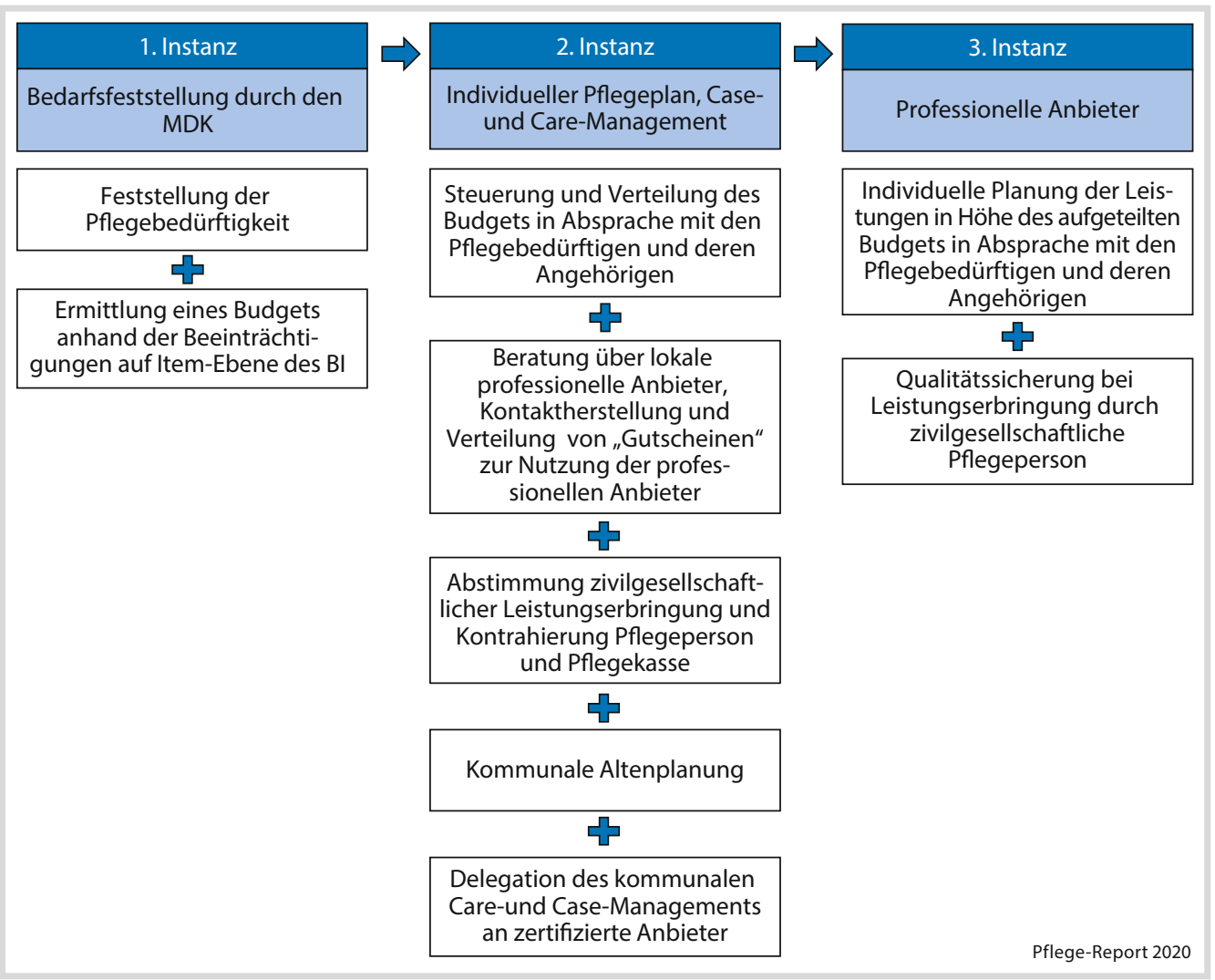

- Abb. 6.1 Institutionelle Ausgestaltung der Bedarfsfeststellung und Pflegeplanung im Reformmodell

angebote auf kommunaler Ebene vorhanden, bekannt und miteinander vernetzt sind.

Im Sinne eines proof of principle wurde dabei gezeigt, dass der Aufbau derartiger Strukturen grundsätzlich möglich ist - ohne dass behauptet wird, das die vorgeschlagenen Lösungen bereits das Ende eines entsprechenden Diskussionsprozesses sein müssen.

Ein zentraler Einwand gegen die Umwandlung der Pflegeversicherung in eine echte Sozialversicherung nach Vorbild der GKV mit individuell bedarfsdeckenden Leistungen (bei betraglich fixem Eigenanteil), ist die Befürchtung, dass dadurch eine moral-hazard-bedingte Überinanspruchnahme in Bezug auf Mengen und/oder Preise ausgelöst werde. Mit dem hier vorgestellten Drei-Instanzen-Modell soll dem entgegengetreten werden: In der ersten Instanz wird der Pflegebedarf fachlich bestimmt - von einem Akteur, der selbst damit keine Gewinnsteigerung vornehmen kann. Die individuelle Pflegeplanung muss sich in der zweiten Instanz dann im Rahmen des vorgegebenen individuellen Budgets bewegen, was einer Leistungsausweitung entgegensteht. Erst in der dritten Instanz kommen Anbieter mit Gewinninteresse zum Zuge - können dann ihren Budgetanteil aber nicht mehr erhöhen. Die Preiskomponente soll durch ein Verfahren gesteuert werden, bei dem zwar die relativen Preise fachlich durch die Aufwandsrelationen bestimmt werden, bei der Festsetzung der Punktwerte auf Landesebene aber wieder die Kostenträger und die Politik einbezogen werden können, um so auch dem Kostenbegrenzungsinteresse Geltung zu verschaffen.

Insgesamt können so die eingangs genannten Geburtsfehler der Pflegeversicherung kor- 
rigiert werden und gleichzeitig auch ein weiteres, seit langem bekanntes Defizit des Pflegesicherungssystems adressiert werden: das bislang unzureichende Case- und Care-Management.

\section{Literatur}

Augurzky B, Borchert L, Deppisch R, Krolop S, Mennicken $\mathrm{R}$, Preuss $M$, Rothgang $H$, Stocker-Müller M, Wasem $J$ (2008) Heimentgelte bei der stationären Pflege in Nordrhein-Westfalen. Ein Bundesländervergleich. RWI-Materialien, Heft 44. Rheinisch-Westfälisches Institut für Wirtschaftsforschung, Essen. https://www. rwi-essen.de/media/content/pages/publikationen/ rwi-materialien/M_44_Pflegekosten-NRW.pdf. Zugegriffen: 15. Apr. 2020

Bartoszek G, Drude C (2015) Pflegen. Grundlagen und Interventionen, 1. Aufl. Urban \& Fischer Elsevier, München

Bundesministerium für Gesundheit (Hrsg) (2019) Konzertierte Aktion Pflege. Vereinbarungstext der Arbeitsgruppen 1 bis 5. https://www. bundesgesundheitsministerium.de/fileadmin/ Dateien/3_Downloads/K/Konzertierte_Aktion Pflege/0619_KAP_Vereinbarungstexte_AG_1-5.pdf. Zugegriffen: 15. Apr. 2020

Bulechek GM, Butcher HK, McCloskey Dochterman J, Wagner CM, Widmer R, Georg J (Hrsg) (2016) Pflegeinterventionsklassifikation (NIC). Hogrefe, Bern (Unter Mitarbeit von Michael Hermann)

DAK (2019) DAK-Gesundheit fordert Reform der Pflegeversicherung. Verwaltungsrat beschließt Resolution zur solidarischen Neuausrichtung. https://www.dak. de/dak/bundesthemen/dak-gesundheit-fordertreform-der-pflegeversicherung-2090932.html. Zugegriffen: 15. Apr. 2020

Geyer J, Korfhage T, Schulz E (2016) Andere Länder, andere Wege: Pflege im internationalen Vergleich [Other countries, other ways: care by international comparison. GuS 70(1):52-58. https://doi.org/10.5771/16115821-2016-1-52

Gori C, Morciano M (2019) Cash-for-care payments in Europe: changes in resource allocation. Soc Policy Adm 53(4):537-550. https://doi.org/10.1111/spol.12508

Heintze C (2015) Auf der Highroad - der skandinavische Weg zu einem zeitgemäßen Pflegesystem. Ein Vergleich zwischen fünf nordischen Ländern und Deutschland. Expertise im Auftrag der Abteilung Wirtschafts- und Sozialpolitik der Friedrich-EbertStiftung, 2. Aufl. WISO Diskurs Expertisen und Dokumentationen zur Wirtschafts- und Sozialpolitik. Friedrich-Ebert-Stiftung, Bonn
Kirschnick O (2016) Pflegetechniken von A-Z, 5. Aufl. Thieme, Stuttgart, New York (Unter Mitarbeit von Doris Kirschnick)

MDS/GKV (Medizinischer Dienst des Spitzenverbandes Bund der Krankenkassen e. V./Spitzenverband Bund der Krankenkassen) (Hrsg) (2017) Richtlinien des GKV-Spitzenverbandes zur Feststellung der Pflegebedürftigkeit nach dem XI. Buch des Sozialgesetzbuches. 2. Aktualisierte Auflage. https://www. mds-ev.de/uploads/media/downloads/17-07-17_ BRi_Pflege.pdf. Zugegriffen: 15. Apr. 2020

Rothgang H (2020) Pflege als „Teilkaskosystem“? Zur richtig verstandenen Bedeutung von Verantwortungsteilung in der Pflege. In: Zerth J (Hrsg) PflegePerspektiven. Ordnungspolitische Aspekte, Erkenntnisse aus der Versorgungsforschung und Implikationen für eine gute Praxis der Pflege. Medhochzwei, Heidelberg (im Erscheinen)

Rothgang H, Kalwitzki T (2017) Alternative Ausgestaltung der Pflegeversicherung - Abbau der Sektorengrenzen und bedarfsgerechte Leistungsstruktur. Gutachten im Auftrag der Initiative Pro-Pflegereform. https://www.pro-pflegereform. de/fileadmin/default/user_upload/Gutachten_ Rothgang_Kalwitzki_-_Alternative_Ausgestaltung_ der_Pflegeversicherung.pdf. Zugegriffen: 16. Apr. 2020

Rothgang H, Kalwitzki T (2019) Sockel-Spitze-Tausch sichert Lebensstandard von Pflegebedürftigen. Neue Caritas 1:2-4

Rothgang H, Fünfstück M, Neubert L, Czwikla J, Hasseler M (2015) Versorgungsaufwände in stationären Pflegeeinrichtungen. Schriftenreihe Modellprogramm zur Weiterentwicklung der Pflegeversicherung, Band 13. GKV-Spitzenverband, Berlin. https://www. gkv-spitzenverband.de/media/dokumente/presse/ publikationen/schriftenreihe/GKV_Schriftenreihe_ Pflege_Band_13.pdf. Zugegriffen: 16. Apr. 2020

Rothgang H, Kalwitzki T, Cordes J (2019) Alternative Ausgestaltung der Pflegeversicherung. 2. Gutachten. https://www.pro-pflegereform. de/fileadmin/default/Gutachten/2._Gutachten_ AAPV_-_Langfassung.pdf. Zugegriffen: 16. Apr. 2020

Rothgang H, Kalwitzki T, Cordes J (2020) Alternative Ausgestaltung der Pflegeversicherung. Die Schwester/ der Pfleg 3:12-17

Rothgang H, Kalwitzki T (2018) Skizze einer neuen Finanzierung der Pflegeversicherung. GesundheitsSozialpolitik - Zeitschrift Für Das Gesamte Gesundheitswes 6:6-12. https://doi.org/10.5771/16115821-2018-6-6

Schneekloth U, Geiss S, Pupeter M, Rothgang H, Kalwitzki T, Müller R (2017) Studie zur Wirkung des Pflege-Neuausrichtungs-Gesetzes (PNG) und des ersten Pflegestärkungsgesetzes (PSG I). Abschlussbericht, Bundesministerium für Gesundheit. TNS Infratest, München. 
https://www.bundesgesundheitsministerium. de/fileadmin/Dateien/5_Publikationen/Pflege/ Berichte/Abschlussbericht_Evaluation_PNG_PSG_ I.pdf. Zugegriffen: 16. Apr. 2020

Schwinger A, Tsiasioti C (2020) Zur Organisations- und Finanzierungszuständigkeit von häuslicher Krankenpflege (SGB V) und medizinischer Behandlungspflege (SGB XI). In: Jacobs K, Kuhlmey A, Greß S, Klauber J, Schwinger A (Hrsg) Pflege-Report 2020. Springer, Berlin Heidelberg

Spasova S, Baeten R, Coster S, Ghailani D, Pena-Casas R, Vernhercke B (2018) Challenges in long-term care in Europe. A study of national policies. European Social Policy Network (ESPN). European Comission, Brussels. https://ec.europa.eu/social/main.jsp? catld $=738$ \&langld $=$ en\&publd $=8128 \&$ furtherPubs $=$ yes. Zugegriffen: 16. Apr. 2020

TRISAN - Trinationales Kompetenzzentrum für ihre Gesundheitsprojekte (Hrsg) (2018) Die Pflege älterer Personen in Deutschland, Frankreich und der Schweiz. https://www.trisan.org/aktuelles/ themenheft-die-pflege-aelterer-personen-indeutschland-frankreich-und-der-schweiz-online/. Zugegriffen: 16. Apr. 2020

Wingenfeld K, Büscher A (2017) Strukturierung und Beschreibung pflegerischer Aufgaben auf der Grundlage des neuen Pflegebedürftigkeitsbegriffs. https://www.bundesgesundheitsministerium. de/fileadmin/Dateien/5_Publikationen/Pflege/ Berichte/Fachbericht_Pflege.pdf. Zugegriffen: 16. Apr. 2020

Wingenfeld K, Büscher A, Gansweid B (2011) Das neue Begutachtungsinstrument zur Feststellung von Pflegebedürftigkeit. Schriftenreihe Modellprogramm zur Weiterentwicklung der Pflegeversicherung, Bd 2. CW Haarfeld, Hürth. https://www.gkvspitzenverband.de/media/dokumente/presse/ publikationen/schriftenreihe/GKV-Schriftenreihe_ Pflege_Band_2_18962.pdf. Zugegriffen: 16. Apr. 2020

Open Access Dieses Kapitel wird unter der Creative Commons Namensnennung 4.0 International Lizenz (http:// creativecommons.org/licenses/by/4.0/deed.de) veröffentlicht, welche die Nutzung, Vervielfältigung, Bearbeitung, Verbreitung und Wiedergabe in jeglichem Medium und Format erlaubt, sofern Sie den/die ursprünglichen Autor(en) und die Quelle ordnungsgemäß nennen, einen Link zur Creative Commons Lizenz beifügen und angeben, ob Änderungen vorgenommen wurden.

Die in diesem Kapitel enthaltenen Bilder und sonstiges Drittmaterial unterliegen ebenfalls der genannten Creative Commons Lizenz, sofern sich aus der Abbildungslegende nichts anderes ergibt. Sofern das betreffende Material nicht unter der genannten Creative Commons Lizenz steht und die betreffende Handlung nicht nach gesetzlichen Vorschriften erlaubt ist, ist für die oben aufgeführten Weiterverwendungen des Materials die Einwilligung des jeweiligen Rechteinhabers einzuholen. 